\title{
ON OPTIMALITY AND DUALITY FOR GENERALIZED NONDIFFERENTIABLE FRACTIONAL OPTIMIZATION PROBLEMS
}

\author{
Moon Hee Kim and Gwi Soo Kim
}

\begin{abstract}
A generalized nondifferentiable fractional optimization problem (GFP), which consists of a maximum objective function defined by finite fractional functions with differentiable functions and support functions, and a constraint set defined by differentiable functions, is considered. Recently, Kim et al. [Journal of Optimization Theory and Applications 129 (2006), no. 1, 131-146] proved optimality theorems and duality theorems for a nondifferentiable multiobjective fractional programming problem (MFP), which consists of a vector-valued function whose components are fractional functions with differentiable functions and support functions, and a constraint set defined by differentiable functions. In fact if $\bar{x}$ is a solution of (GFP), then $\bar{x}$ is a weakly efficient solution of (MFP), but the converse may not be true. So, it seems to be not trivial that we apply the approach of Kim et al. to (GFP). However, modifying their approach, we obtain optimality conditions and duality results for (GFP).
\end{abstract}

\section{Introduction}

Many authors have introduced various concepts of generalized convexity and have obtained duality results for a fractional programming problem ([2]-[8], [11]). In [4], Kuk et al. defined the concept of $(V, \rho)$-invexity for vector-valued functions, which is generalization of the $V$-invexity concept; they proved the generalized Karush-Kuhn-Tucker sufficient optimality theorem as well as weak and strong duality for nonsmooth multi-objective programs under the $(V, \rho)$ invexity assumptions. Later, Kuk et al. [5] extended their results to nonsmooth multiobjective fractional programs.

In 1996, Mond and Schechter [10] obtained duality and optimality for nondifferentiable multiobjective programming problems in which the objective function contains a support function.

Received April 22, 2009; Revised September 14, 2009.

2000 Mathematics Subject Classification. Primary 90C26, 90C30, 90C46.

Key words and phrases. fractional optimization problem, weakly efficient solution, optimality condition, duality.

This work was supported by the Korea Science and Engineering Foundation (KOSEF) NRL program grant funded by the Korea government(MEST)(No. ROA-2008-000-20010-0). 
Recently, Kim et al. [2] proved optimality theorem and duality theorem for a nondifferentiable multiobjective fractional programming problem (MFP), which consists of a vector-valued function whose components are fractional functions with differentiable functions and support functions, and a constraint set defined by differentiable functions.

Now we consider the following generalized fractional problem (GFP):

$$
\begin{array}{lll}
(\mathrm{GFP}) & \text { Minimize } & \max \left\{\frac{f_{i}(x)+s\left(x \mid C_{i}\right)}{g_{i}(x)} \mid i=1, \ldots, p\right\} \\
\text { subject to } & h_{j}(x) \leq 0, \quad j=1, \ldots, m,
\end{array}
$$

where $f:=\left(f_{1}, \ldots, f_{p}\right): \mathbb{R}^{n} \rightarrow \mathbb{R}^{p}, g:=\left(g_{1}, \ldots, g_{p}\right): \mathbb{R}^{n} \rightarrow \mathbb{R}^{p}$ and $h:=$ $\left(h_{1}, \ldots, h_{m}\right): \mathbb{R}^{n} \rightarrow \mathbb{R}^{m}$ are continuously differentiable. We assume that $g_{i}(x)>0, i=1, \ldots, p$. For each $i=1, \ldots, p, C_{i}$ is a compact convex set of $\mathbb{R}^{n}$ and we define a support function with respect to $C_{i}$ as follows:

$$
s\left(x \mid C_{i}\right):=\max \left\{\left\langle x, y_{i}\right\rangle \mid y_{i} \in C_{i}\right\} .
$$

Further let, $J(x)=\left\{j: h_{j}(x)=0\right\}$, for any $x \in \mathbb{R}^{n}$ and let

$$
k_{i}(x)=s\left(x \mid C_{i}\right), \quad i=1, \ldots, p .
$$

Then, $k_{i}$ is a convex function and we can prove that

$$
\partial k_{i}(x)=\left\{w_{i} \in C_{i} \mid\left\langle w_{i}, x\right\rangle=s\left(x \mid C_{i}\right)\right\},
$$

where $\partial k_{i}$ is the subdifferential of $k_{i}$.

We recall the nondifferentaible multiobjective fractional programming problem $(\mathrm{MFP})$ in [2]:

$$
\begin{array}{rll}
(\mathrm{MFP}) & \text { Minimize } & \left(\frac{f_{1}(x)+s\left(x \mid C_{1}\right)}{g_{1}(x)}, \ldots, \frac{f_{p}(x)+s\left(x \mid C_{p}\right)}{g_{p}(x)}\right) \\
\text { subject to } & h_{j}(x) \leq 0, \quad j=1, \ldots, m,
\end{array}
$$

where $f:=\left(f_{1}, \ldots, f_{p}\right): \mathbb{R}^{n} \rightarrow \mathbb{R}^{p}, g:=\left(g_{1}, \ldots, g_{p}\right): \mathbb{R}^{n} \rightarrow \mathbb{R}^{p}$ and $h:=$ $\left(h_{1}, \ldots, h_{m}\right): \mathbb{R}^{n} \rightarrow \mathbb{R}^{m}$ are continuously differentiable. We assume that $g_{i}(x)>0, i=1, \ldots, p$. Further let, $S=\left\{x \in \mathbb{R}^{n} \mid h_{j}(x) \leq 0, j=1, \ldots, m\right\}$.

Weakly efficient solution of (MFP) are defined as follows:

Definition. A point $\bar{x} \in S$ is a weakly efficient solution of (MFP) if there exist no other feasible point $x \in S$ such that $\frac{f_{i}(x)+s\left(x \mid C_{i}\right)}{g_{i}(x)}<\frac{f_{i}(\bar{x})+s\left(\bar{x} \mid C_{i}\right)}{g_{i}(\bar{x})}$ for all $i=1,2, \ldots, p$.

Then $\operatorname{sol}(\mathrm{GFP}) \subset W E f f(\mathrm{MFP})$, where $\operatorname{sol}(\mathrm{GFP})$ is the set of all minimum of (GFP) and $W E f f(\mathrm{MFP})$ is the set of all weakly efficient solution of (MFP). But the converse may not be true.

Example 1.1. Let $f_{1}(x)=x, f_{2}(x)=x^{2}, C_{1}=C_{2}=\{0\}, g_{1}(x)=g_{2}(x)=1$ and $h(x)=x$. Then $\operatorname{sol}(\mathrm{GFP})=\{0\}$ and $W E f f(\mathrm{MFP})=(-\infty, 0]$. 
The above example says that the inclusion: $W E f f(\mathrm{MFP}) \subset \operatorname{sol}(\mathrm{GFP})$ may not be true. So, it seems to be not trivial that we apply the approach of Kim et al. [2] to (GFP). However, in this paper, we can modifying their approach, we obtain optimality conditions and duality results for (GFP).

We introduce the following definition due to Kuk et al. [4].

Definition. A vector function $f: \mathbb{R}^{n} \rightarrow \mathbb{R}^{p}$ is said to be $(V, \rho)$-invex at $u \in \mathbb{R}^{n}$ with respect to the functions $\eta$ and $\theta_{i}: \mathbb{R}^{n} \times \mathbb{R}^{n} \rightarrow \mathbb{R}^{n}$ if there exists $\alpha_{i}: \mathbb{R}^{n} \times \mathbb{R}^{n} \rightarrow \mathbb{R}_{+} \backslash\{0\}$ and $\rho_{i} \in \mathbb{R}, i=1, \ldots, p$ such that for any $x \in \mathbb{R}^{n}$ and for all $i=1, \ldots, p$,

$$
\alpha_{i}(x, u)\left[f_{i}(x)-f_{i}(u)\right] \geq \nabla f_{i}(u) \eta(x, u)+\rho_{i}\left\|\theta_{i}(x, u)\right\|^{2} .
$$

Definition. A vector function $f: \mathbb{R}^{n} \rightarrow \mathbb{R}^{p}$ is said to be $\eta$-invex at $u \in \mathbb{R}^{n}$ such that for any $x \in \mathbb{R}^{n}$ and for all $i=1, \ldots, p$,

$$
f_{i}(x)-f_{i}(u) \geq \nabla f_{i}(u) \eta(x, u) .
$$

We recall the following theorem due to Kim et al. [2]

Theorem 1.1. Assume that $f$ and $g$ are vector-valued differentiable functions defined on $\mathbb{R}^{n}$ and $f(x)+\langle w, x\rangle \geq 0, g(x)>0$ for all $x \in \mathbb{R}^{n}$. If $f(\cdot)+\langle w, \cdot\rangle$ and $-g(\cdot)$ are $(V, \rho)$-invex at $x_{0}$, then $\frac{f(\cdot)+\langle w, \cdot\rangle}{g(\cdot)}$ is $(V, \rho)$-invex at $x_{0}$, where

$$
\bar{\alpha}_{i}\left(x, x_{0}\right)=\frac{g_{i}(x)}{g_{i}\left(x_{0}\right)} \alpha_{i}\left(x, x_{0}\right), \quad \bar{\theta}_{i}\left(x, x_{0}\right)=\left(\frac{1}{g_{i}\left(x_{0}\right)}\right)^{\frac{1}{2}} \theta\left(x, x_{0}\right),
$$

that is, for all $i$,

$$
\begin{aligned}
& \alpha_{i}\left(x, x_{0}\right)\left[\frac{f_{i}(x)+\left\langle w_{i}, x\right\rangle}{g_{i}(x)}-\frac{f_{i}\left(x_{0}\right)+\left\langle w_{i}, x_{0}\right\rangle}{g_{i}\left(x_{0}\right)}\right] \\
\geq & \frac{g_{i}\left(x_{0}\right)}{g_{i}(x)}\left[\nabla\left(\frac{f_{i}\left(x_{0}\right)+\left\langle w_{i}, x_{0}\right\rangle}{g_{i}\left(x_{0}\right)}\right) \eta\left(x, x_{0}\right)+\rho_{i}\left\|\left(\frac{1}{g_{i}\left(x_{0}\right)}\right)^{\frac{1}{2}} \theta_{i}\left(x, x_{0}\right)\right\|^{2}\right] .
\end{aligned}
$$

\section{Optimality conditions}

Now, we establish the Kuhn-Tucker necessary and sufficient conditions for a solution of (GFP).

Theorem 2.1 (Kuhn-Tucker Necessary Optimality Theorem). If $x_{0}$ is a solution of $(G F P)$, and assume that $0 \notin c o\left\{\nabla h_{j}\left(x_{0}\right) \mid j \in J\left(x_{0}\right)\right\}$, then there exist $\lambda_{i} \geq 0, i \in I\left(x_{0}\right):=\left\{i \mid \max \left\{\frac{f_{i}\left(x_{0}\right)+s\left(x_{0} \mid C_{i}\right)}{g_{i}\left(x_{0}\right)} \mid i=1, \ldots, p\right\}\right\}$, 
$\sum_{i \in I\left(x_{0}\right)} \lambda_{i}=1, \mu_{j} \geq 0, j=1, \ldots, m$ and $w_{i} \in C_{i}, i \in I\left(x_{0}\right)$ such that

$$
\begin{aligned}
& \sum_{i \in I\left(x_{0}\right)} \lambda_{i} \nabla\left(\frac{f_{i}\left(x_{0}\right)+\left\langle w_{i}, x_{0}\right\rangle}{g_{i}\left(x_{0}\right)}\right)+\sum_{j=1}^{m} \mu_{j} \nabla h_{j}\left(x_{0}\right)=0, \\
& \left\langle w_{i}, x_{0}\right\rangle=s\left(x_{0} \mid C_{i}\right), \\
& \sum_{j=1}^{m} \mu_{j} h_{j}\left(x_{0}\right)=0 .
\end{aligned}
$$

Proof. Let $\varphi_{i}(x)=\frac{f_{i}(x)+s\left(x \mid C_{i}\right)}{g_{i}(x)}, i=1, \ldots, p$. Let $x_{0}$ be a solution of (GFP) and let $I\left(x_{0}\right)=\left\{i \mid \max \left\{\varphi_{i}\left(x_{0}\right) \mid i=1, \ldots, p\right\}\right\}$. Then by Proposition 2.3.12 in [1] and Corollary 5.1.8 in [9], there exist $\mu_{j} \geq 0, j=1, \ldots, m$,

$$
\begin{aligned}
& 0 \in \operatorname{co}\left\{\partial^{c} \varphi_{i}\left(x_{0}\right) \mid i \in I\left(x_{0}\right)\right\}+\sum_{j=1}^{m} \mu_{j} \partial^{c} h_{j}\left(x_{0}\right) \\
& \text { and } \mu_{j} h_{j}\left(x_{0}\right)=0
\end{aligned}
$$

Thus there exist $\lambda_{i} \geq 0, i \in I\left(x_{0}\right), \sum_{i \in I\left(x_{0}\right)} \lambda_{i}=1$ such that

$$
\begin{aligned}
& 0 \in \sum_{i \in I\left(x_{0}\right)} \lambda_{i} \partial^{c} \varphi_{i}\left(x_{0}\right)+\sum_{j=1}^{m} \mu_{j} \nabla h_{j}\left(x_{0}\right) \\
& \text { and } \mu_{j} h_{j}\left(x_{0}\right)=0 .
\end{aligned}
$$

By Proposition 2.3.14 in [1],

$$
\partial^{c} \varphi_{i}\left(x_{0}\right)=\frac{g_{i}\left(x_{0}\right)\left(\nabla f_{i}\left(x_{0}\right)+\partial s\left(x_{0} \mid C_{i}\right)\right)-\left(f_{i}\left(x_{0}\right)+s\left(x_{0} \mid C_{i}\right)\right) \nabla g_{i}\left(x_{0}\right)}{g_{i}^{2}\left(x_{0}\right)} .
$$

Since

$$
\begin{aligned}
\partial^{c} \varphi_{i}\left(x_{0}\right) & =\left\{\frac{g_{i}\left(x_{0}\right)\left(\nabla f_{i}\left(x_{0}\right)+w_{i}\right)-\left(f_{i}\left(x_{0}\right)+\left\langle w_{i}, x_{0}\right\rangle\right) \nabla g_{i}\left(x_{0}\right)}{g_{i}^{2}\left(x_{0}\right)} \mid w_{i} \in C_{i},\right. \\
\left.\left\langle w_{i}, x_{0}\right\rangle=s\left(x_{0} \mid C_{i}\right), i \in I\left(x_{0}\right)\right\} & \\
& =\left\{\nabla\left(\frac{f_{i}\left(x_{0}\right)+\left\langle w_{i}, x_{0}\right\rangle}{g_{i}\left(x_{0}\right)}\right) \mid w_{i} \in C_{i},\left\langle w_{i}, x_{0}\right\rangle=s\left(x_{0} \mid C_{i}\right), i \in I\left(x_{0}\right)\right\}
\end{aligned}
$$

and hence from (2.1), there exist $\lambda_{i} \geq 0, i \in I\left(x_{0}\right), \sum_{i \in I\left(x_{0}\right)} \lambda_{i}=1, \mu_{j} \geq$ $0, j=1, \ldots, m$ and $w_{i} \in C_{i}, i \in I\left(x_{0}\right)$ such that

$$
\begin{aligned}
& \sum_{i \in I\left(x_{0}\right)} \lambda_{i} \nabla\left(\frac{f_{i}\left(x_{0}\right)+\left\langle w_{i}, x_{0}\right\rangle}{g_{i}\left(x_{0}\right)}\right)+\sum_{j=1}^{m} \mu_{j} \nabla h_{j}\left(x_{0}\right)=0, \\
& \left\langle w_{i}, x_{0}\right\rangle=s\left(x_{0} \mid C_{i}\right) \\
& \sum_{j=1}^{m} \mu_{j} h_{j}\left(x_{0}\right)=0 .
\end{aligned}
$$


Theorem 2.2 (Kuhn-Tucker Sufficient Optimality Theorem). Let $x_{0}$ be a feasible solution of $(G F P)$. Suppose that there exist $\lambda_{i} \geq 0, i \in I\left(x_{0}\right)$, $\sum_{i \in I\left(x_{0}\right)} \lambda_{i}=1, \mu_{j} \geq 0, j=1, \ldots, m$ and $w_{i} \in C_{i}, i \in I\left(x_{0}\right)$ such that

$$
\begin{aligned}
& \sum_{i \in I\left(x_{0}\right)} \lambda_{i} \nabla\left(\frac{f_{i}\left(x_{0}\right)+\left\langle w_{i}, x_{0}\right\rangle}{g_{i}\left(x_{0}\right)}\right)+\sum_{j=1}^{m} \mu_{j} \nabla h_{j}\left(x_{0}\right)=0, \\
& \left\langle w_{i}, x_{0}\right\rangle=s\left(x_{0} \mid C_{i}\right), \\
& \sum_{j=1}^{m} \mu_{j} h_{j}\left(x_{0}\right)=0 .
\end{aligned}
$$

If $f(\cdot)+\langle w, \cdot\rangle$ and $-g(\cdot)$ are $(V, \rho)$-invex at $x_{0}$, and $h$ is $\eta$-invex at $x_{0}$ with respect to the same $\eta$, and $\sum_{i \in I\left(x_{0}\right)} \lambda_{i} \rho_{i}\left\|\bar{\theta}_{i}\left(x, x_{0}\right)\right\|^{2} \geq 0$, then $x_{0}$ is a solution of $(G F P)$.

Proof. Suppose that $x_{0}$ is not a solution of (GFP). Then there exist a feasible solution $x$ of (GFP) such that

$$
\max _{1 \leq i \leq p} \frac{f_{i}(x)+s\left(x \mid C_{i}\right)}{g_{i}(x)}<\max _{1 \leq i \leq p} \frac{f_{i}\left(x_{0}\right)+s\left(x_{0} \mid C_{i}\right)}{g_{i}\left(x_{0}\right)} .
$$

Then

$$
\frac{f_{i}(x)+s\left(x \mid C_{i}\right)}{g_{i}(x)}<\frac{f_{i}\left(x_{0}\right)+s\left(x_{0} \mid C_{i}\right)}{g_{i}\left(x_{0}\right)} \text { for all } i \in I\left(x_{0}\right) .
$$

Since $\left\langle w_{i}, x_{0}\right\rangle=s\left(x_{0} \mid C_{i}\right)$ and $w_{i} \in C_{i}$, we have for all $i \in I\left(x_{0}\right)$,

$$
\begin{aligned}
\frac{f_{i}(x)+\left\langle w_{i}, x\right\rangle}{g_{i}(x)} & \leq \frac{f_{i}(x)+s\left(x \mid C_{i}\right)}{g_{i}(x)} \\
& <\frac{f_{i}\left(x_{0}\right)+s\left(x_{0} \mid C_{i}\right)}{g_{i}\left(x_{0}\right)} \\
& =\frac{f_{i}\left(x_{0}\right)+\left\langle w_{i}, x_{0}\right\rangle}{g_{i}\left(x_{0}\right)}
\end{aligned}
$$

and hence $\bar{\alpha}_{i}\left(x, x_{0}\right)>0$,

$$
\bar{\alpha}_{i}\left(x, x_{0}\right)\left[\frac{f_{i}(x)+\left\langle w_{i}, x\right\rangle}{g_{i}(x)}-\frac{f_{i}\left(x_{0}\right)+\left\langle w_{i}, x_{0}\right\rangle}{g_{i}\left(x_{0}\right)}\right]<0 .
$$

By the $(V, \rho)$-invexity of $f(\cdot)+\langle w, \cdot\rangle$ and $-g(\cdot)$ at $x_{0}$, and by Theorem 1.1 , we have

$$
\nabla\left(\frac{f_{i}\left(x_{0}\right)+\left\langle w_{i}, x_{0}\right\rangle}{g_{i}\left(x_{0}\right)}\right) \eta\left(x, x_{0}\right)+\rho_{i}\left\|\bar{\theta}_{i}\left(x, x_{0}\right)\right\|^{2}<0 .
$$

Hence, we have

$$
\sum_{i \in I\left(x_{0}\right)} \lambda_{i} \nabla\left(\frac{f_{i}\left(x_{0}\right)+\left\langle w_{i}, x_{0}\right\rangle}{g_{i}\left(x_{0}\right)}\right) \eta\left(x, x_{0}\right)+\sum_{i \in I\left(x_{0}\right)} \lambda_{i} \rho_{i}\left\|\bar{\theta}_{i}\left(x, x_{0}\right)\right\|^{2}<0 .
$$


Since $\sum_{i \in I\left(x_{0}\right)} \lambda_{i} \rho_{i}\left\|\bar{\theta}_{i}\left(x, x_{0}\right)\right\|^{2} \geq 0$

$$
\sum_{i \in I\left(x_{0}\right)} \lambda_{i} \nabla\left(\frac{f_{i}\left(x_{0}\right)+\left\langle w_{i}, x_{0}\right\rangle}{g_{i}\left(x_{0}\right)}\right) \eta\left(x, x_{0}\right)<0
$$

and so, it follows from (2.2) that

$$
\sum_{j=1}^{m} \mu_{j} \nabla h_{j}\left(x_{0}\right) \eta\left(x, x_{0}\right)>0 \text {. }
$$

Then, by the $\eta$-invexity of $h$, we have

$$
\sum_{j=1}^{m} \mu_{j} h_{j}(x)-\sum_{j=1}^{m} \mu_{j} h_{j}\left(x_{0}\right)>0 .
$$

Since $\sum_{j=1}^{m} \mu_{j} h_{j}\left(x_{0}\right)=0$, we have $\sum_{j=1}^{m} \mu_{j} h_{j}(x)>0$, which is a contradiction since $\mu_{j} \geq 0, j=1, \ldots, m$ and $x$ is a feasible solution of (GFP). Consequently, $x_{0}$ is a solution of (GFP).

\section{Duality theorems}

Now, we propose the following Mond-Weir type dual problem (DGFP):

(DGFP) Maximize $\max \left\{\frac{f_{i}(u)+s\left(u \mid C_{i}\right)}{g_{i}(u)} \mid i=1, \ldots, p\right\}$

$$
\begin{array}{ll}
\text { subject to } & \sum_{i \in I(u)} \lambda_{i} \nabla\left(\frac{f_{i}(u)+\left\langle w_{i}, u\right\rangle}{g_{i}(u)}\right)+\sum_{j=1}^{m} \mu_{j} \nabla h_{j}(u)=0, \\
& w_{i} \in C_{i},\left\langle w_{i}, u\right\rangle=s\left(u \mid C_{i}\right), i \in I(u) \\
& \sum_{j=1}^{m} \mu_{j} h_{j}(u)=0 \\
& \lambda_{i} \geq 0, i \in I(u), \sum_{i \in I(u)} \lambda_{i}=1, \mu_{j} \geq 0, j=1, \ldots, m .
\end{array}
$$

Now we show that the following weak duality theorem holds between (GFP) and (DGFP).

Theorem 3.1 (Weak Duality). Let $x$ be a feasible for $(G F P)$ and let $(u, \lambda, \mu$, $w)$ be feasible for $(D G F P)$. Assume that $f(\cdot)+\langle w, \cdot\rangle$ and $-g(\cdot)$ are $(V, \rho)$-invex at $u$, and let $h$ is $\eta$-invex at $u$ with respect to the same $\eta$, and

$$
\sum_{i \in I(u)} \lambda_{i} \rho_{i}\left\|\bar{\theta}_{i}(x, u)\right\|^{2} \geq 0 .
$$

Then the following holds:

$\max \left\{\frac{f_{i}(x)+s\left(x \mid C_{i}\right)}{g_{i}(x)} \mid i=1, \ldots, p\right\} \geq \max \left\{\frac{f_{i}(u)+s\left(u \mid C_{i}\right)}{g_{i}(u)} \mid i=1, \ldots, p\right\}$. 
Proof. Let $x$ be any feasible for (GFP) and let $(u, \lambda, \mu, w)$ be any feasible for (DGFP). Then we have

$$
\sum_{j=1}^{m} \mu_{j} h_{j}(x) \leq 0 \leq \sum_{j=1}^{m} \mu_{j} h_{j}(u)
$$

By the $\eta$-invexity of $h_{j}(u), j=1, \ldots, m$, we have

$$
\sum_{j=1}^{m} \mu_{j} \nabla h_{j}(u) \eta(x, u) \leq 0 .
$$

Using (3.1), we obtain

$$
\sum_{i \in I(u)} \lambda_{i} \nabla\left(\frac{f_{i}(u)+\left\langle w_{i}, u\right\rangle}{g_{i}(u)}\right) \eta(x, u) \geq 0 .
$$

Now suppose that

$\max \left\{\frac{f_{i}(x)+s\left(x \mid C_{i}\right)}{g_{i}(x)} \mid i=1, \ldots, p\right\}<\max \left\{\frac{f_{i}(u)+s\left(u \mid C_{i}\right)}{g_{i}(u)} \mid i=1, \ldots, p\right\}$.

Then

$$
\frac{f_{i}(x)+s\left(x \mid C_{i}\right)}{g_{i}(x)}<\frac{f_{i}(u)+s\left(u \mid C_{i}\right)}{g_{i}(u)} \text { for all } i \in I(u) .
$$

Since $\left\langle w_{i}, u\right\rangle=s\left(u \mid C_{i}\right)$, we have for all $i \in I(u)$,

$$
\frac{f_{i}(x)+\left\langle w_{i}, x\right\rangle}{g_{i}(x)}<\frac{f_{i}(u)+\left\langle w_{i}, u\right\rangle}{g_{i}(u)} .
$$

By Theorem 1.1, we have,

$$
\begin{aligned}
0 & >\bar{\alpha}_{i}(x, u)\left[\frac{f_{i}(x)+\left\langle w_{i}, x\right\rangle}{g_{i}(x)}-\frac{f_{i}(u)+\left\langle w_{i}, u\right\rangle}{g_{i}(u)}\right] \\
& \geq \nabla\left(\frac{f_{i}(u)+\left\langle w_{i}, u\right\rangle}{g_{i}(u)}\right) \eta(x, u)+\rho_{i}\left\|\bar{\theta}_{i}(x, u)\right\|^{2} .
\end{aligned}
$$

By using $\lambda_{i} \geq 0, i \in I(u)$, we have,

$$
\sum_{i \in I(u)} \lambda_{i} \nabla\left(\frac{f_{i}(u)+\left\langle w_{i}, u\right\rangle}{g_{i}(u)}\right) \eta(x, u)+\sum_{i \in I(u)} \lambda_{i} \rho_{i}\left\|\bar{\theta}_{i}(x, u)\right\|^{2}<0 .
$$

Since $\sum_{i \in I(u)} \lambda_{i} \rho_{i}\left\|\bar{\theta}_{i}(x, u)\right\|^{2} \geq 0$, we have

$$
\sum_{i \in I(u)} \lambda_{i} \nabla\left(\frac{f_{i}(u)+\left\langle w_{i}, u\right\rangle}{g_{i}(u)}\right) \eta(x, u)<0,
$$

which contradicts (3.2). Hence the result holds.

Now we give a strong duality theorem which holds between (GFP) and (DGFP). 
Theorem 3.2 (Strong Duality). If $\bar{x}$ be a solution of $(G F P)$ and suppose that $0 \notin \operatorname{co}\left\{\nabla h_{j}(\bar{x}) \mid j \in J(\bar{x})\right\}$. Then there exist $\bar{\lambda} \in \mathbb{R}^{p}, \bar{\mu} \in \mathbb{R}^{m}$ and $\bar{w} \in C$ such that $(\bar{x}, \bar{\lambda}, \bar{\mu}, \bar{w})$ is feasible for $(D G F P)$. Moreover if the weak duality holds, then $(\bar{x}, \bar{\lambda}, \bar{\mu}, \bar{w})$ is a solution of $(D G F P)$.

Proof. By Theorem 2.1, there exist $\bar{\lambda} \in \mathbb{R}^{p}, \bar{\mu} \in \mathbb{R}^{m}$ and $\bar{w}_{i} \in C_{i}, i \in I(\bar{x})$, such that

$$
\begin{aligned}
& \sum_{i \in I(\bar{x})} \bar{\lambda}_{i} \nabla\left(\frac{f_{i}(\bar{x})+\left\langle\bar{w}_{i}, \bar{x}\right\rangle}{g_{i}(\bar{x})}\right)+\sum_{j=1}^{m} \bar{\mu}_{j} \nabla h_{j}(\bar{x})=0, \\
& \left\langle\bar{w}_{i}, \bar{x}\right\rangle=s\left(\bar{x} \mid C_{i}\right), \\
& \sum_{j=1}^{m} \mu_{j} h_{j}(\bar{x})=0, \\
& \lambda_{i} \geq 0, \quad i \in I(\bar{x}), \sum_{i \in I(\bar{x})} \lambda_{i}=1 .
\end{aligned}
$$

Thus $(\bar{x}, \bar{\lambda}, \bar{\mu}, \bar{w})$ is a feasible for (DGFP). On the other hand, by weak duality (Theorem 3.1),

$$
\max \left\{\frac{f_{i}(\bar{x})+s\left(\bar{x} \mid C_{i}\right)}{g_{i}(\bar{x})} \mid i=1, \ldots, p\right\} \geq \max \left\{\frac{f_{i}(u)+s\left(u \mid C_{i}\right)}{g_{i}(u)} \mid i=1, \ldots, p\right\}
$$

for any (DGFP) feasible solution $(u, \lambda, \mu, w)$. Hence $(\bar{x}, \bar{\lambda}, \bar{\mu}, \bar{w})$ is a solution of (DGFP).

\section{References}

[1] F. H. Clarke, Optimization and Nonsmooth Analysis, A Wiley-Interscience Publication, John Wiley \& Sons, 1983

[2] D. S. Kim, S. J. Kim, and M. H. Kim, Optimality and duality for a class of nondifferentiable multiobjective fractional programming problems, Journal of Optimization Theory and Applications 129 (2006), no. 1, 131-146.

[3] M. H. Kim and D. S. Kim, Non-differentiabel symmetric duality for multiobjective programming with cone constraints, European Journal of Operational Research 188 (2008), $652-661$.

[4] H. Kuk, G. M. Lee, and D. S. Kim, nonsmooth multiobjective programs with $(V, \rho)$ invexity, Indian Journal of Pure and Applied Mathematics 29 (1998), 405-412.

[5] H. Kuk, G. M. Lee, and T. Tanino, Optimality and duality for nonsmooth multiobjective fractional programming with generalized invexity, Journal of Mathematical Analysis and Applications 262 (2001), 365-375.

[6] Z. Liang, H. Huang, and P. M. Pardalos, Optimality conditions and duality for a class of nonlinear fractional programming problems, Journal of Optimization Theory and Applications 110 (2001), 611-619.

[7] _ Efficiency conditions and duality for a class of multiobjective fractional programming problems, Journal of Global Optimization 27 (2003), 444-417.

[8] X. J. Long, N. J. Huang, and Z. B. Liu, Optimality conditions, duality ad saddle points for nondifferentiale multiobjective fractional programs, Journal of Industry Management and Optimization 4 (2008), 287-298. 
[9] M. M. Mäklelä and P. Neittaanmäki, Nonsmooth Optimization: Analysis and Algorithms with Applications to Optimal Control, World Scientific Publishing Co. Pte. Ltd. 1992.

[10] B. Mond and M. Schechter, Nondifferentiable symmetric duality, Bulletine of the Australian Mathematical Society 53 (1996), 177-187.

[11] X. M. Yang, X. Q. Yang, and K. L. Teo, Duality and saddle-point type optimality for generalized nonlinear fractional programming, Journal of Mathematical Analysis and Applications 289 (2004), 100-109.

Moon Hee Kim

Department of Multimedia EngineEring

TONGMYONG UNIVERSITY

PUSAN 608-711, Korea

E-mail address: mooni@tu.ac.kr

Gwi Soo KIm

Department of Applied Mathematics

Pukyong National University

Pusan 608-737, Korea

E-mail address: gwisoo1103@hanmail.net 